\title{
Validation of a LC/MS method for the determination of gemfibrozil in human plasma and its application to a pharmacokinetic study
}

\author{
Joseph E. Rower, Lane R. Bushman, Kyle P. Hammond, Rajendra S. Kadam, and Christina \\ L. Aquilante \\ University of Colorado Denver, School of Pharmacy, Department of Pharmaceutical Sciences, \\ Aurora, Colorado.
}

\begin{abstract}
Gemfibrozil, a fibric acid hypolipidemic agent, is increasingly being used in clinical drug-drug interaction studies as an inhibitor of drug metabolizing enzymes and drug transporters. The validation of a fast, accurate, and precise LC/MS method is described for the quantitative determination of gemfibrozil in an EDTA-anticoagulated human plasma matrix. Briefly, gemfibrozil was extracted from human plasma by an acetonitrile protein precipitation method. The assay was reproducible with intra-assay precision between $1.6 \%$ and $10.7 \%$, and inter-assay precision ranging from $4.4 \%$ to $7.8 \%$. The assay also showed good accuracy, with intra-assay concentrations within $85.6 \%$ and $108.7 \%$ of the expected value, and inter-assay concentrations within 89.4 to $104.0 \%$ of the expected value. The linear concentration range was between 0.5 and $50 \mu \mathrm{g} / \mathrm{mL}$ with a lower limit of quantitation of $0.5 \mu \mathrm{g} / \mathrm{mL}$ when $125 \mu \mathrm{L}$ of plasma were extracted. This LC/MS method yielded a quick, simple, and reliable protocol for determining gemfibrozil concentrations in plasma and is applicable to clinical pharmacokinetic studies.
\end{abstract}

\section{Keywords}

gemfibrozil; LC/MS; pharmacokinetic; human plasma

\section{Introduction}

Gemfibrozil (GFZ, Lopid ${ }^{\circledR}$ ) is a fibric acid derivative used to treat dyslipidemia, primarily hypertriglyceridemia (Lopid Prescribing Information, 2008). GFZ and its glucuronide conjugate are potent inhibitors of the cytochrome P450 (CYP) 2C8 metabolizing enzyme (Prueksaritanont et al., 2005; Ogilvie et al., 2006). Clinically, GFZ has been shown to dramatically decrease the metabolism, and increase the plasma exposure, of CYP2C8 substrates such as pioglitazone, rosiglitazone, repaglinide, and cerivastatin (Backman et al., 2002; Niemi et al., 2003; Niemi et al., 2003; Deng et al., 2005; Jaakkola et al., 2005; Tornio et al., 2008). Recently, GFZ has also been identified as an inhibitor of organic aniontransporting polypeptide 1B1 (OATP1B1), a drug transporter located on the basolateral membrane of hepatocytes (Shitara et al., 2004; Yamazaki et al., 2005). GFZ-mediated inhibition of OATP1B1 has been shown to decrease the hepatic uptake, and increase the plasma exposure, of OATP1B1 substrates such as pravastatin, cerivastatin, and rosuvastatin

Corresponding Author: Christina Aquilante, Pharm.D., 12700 East $19^{\text {th }}$ Avenue, P15-C238, Aurora, Colorado 80045; Phone (303) 724-6126; Fax (303) 724-7266; Christina.aquilante@ucdenver.edu.

Conflict of Interest: None to declare. 
(Shitara et al., 2004; Schneck et al., 2004; Nakagomi-Hagihara et al., 2007). Given its pharmacology as a CYP2C8 and OATP1B1 inhibitor, GFZ is being used more often in clinical drug-drug interaction studies to test the effects of CYP2C8 or OATP1B1 inhibition on substrate pharmacokinetics. Thus, simple, sensitive, and cost effective analytical methods for the determination of GFZ concentrations in human plasma are becoming increasingly important in the field of clinical pharmacology.

To date, the majority of papers reporting bioanalytical methods for the quantification of GFZ in plasma have used HPLC-UV (sensitivities ranging from $0.05 \mu \mathrm{g} / \mathrm{ml}$ to $1 \mu \mathrm{g} / \mathrm{ml}$ ) and HPLC-fluorescence (sensitivities ranging from $0.01 \mu \mathrm{g} / \mathrm{ml}$ to $0.5 \mu \mathrm{g} / \mathrm{ml}$ ) (Hengy et al., 1985; Randinitis et al., 1986; Forland et al., 1987; Nakagawa et al., 1991; Hermening et al., 2000; Gonzalez-Penas et al., 2001; Niemi et al., 2003; Schneck et al., 2004; Deng et al., 2005; Vittal et al., 2006; Kang et al., 2009). Fluorescence and UV detection methods have long run times (in some cases up to 20 minutes), and require extensive sample preparation techniques (Forland et al., 1987; Nakagawa et al., 1991; Hermening et al., 2000; Vittal et al., 2006; Kang et al., 2009). Tandem mass spectrometry (LC-MS/MS) bioanalytical methods have been reported for GFZ, with assay sensitivities ranging from $0.001 \mu \mathrm{g} / \mathrm{ml}$ to $0.1 \mu \mathrm{g} / \mathrm{ml}$ and shorter run times (e.g., 2 minutes) (Roadcap et al., 2003; Tornio et al., 2008; Busse et al., 2009). While LC-MS/MS has the potential to produce the most sensitive and specific analytical methods, the instrumentation and maintenance are very expensive, require a high-level of technical expertise, and are not feasible for all laboratories (McIntosh et al., 2007). Single quadrupole mass spectrometry (LC/MS) is a useful, albeit underutilized, alternative to LC-MS/MS (McIntosh et al., 2007). LC/MS requires fewer resources than LC-MS/MS, is relatively easy to use, and can provide sensitivities that mirror those observed with HPLC-UV and HPLC-fluorescence methods. In addition, the use of LC/MS increases assay specificity in comparison to UV and fluorescence detections, as analyte separation can be done with both MS (i.e., monitoring a single mass to charge ratio) and HPLC (i.e., by looking at specific analyte retention times). In contrast, UV and fluorescence methods rely on separation of target analytes via HPLC before detection. Thus, LC/MS provides the advantage of being able to differentiate co-eluting compounds. To our knowledge, the validation of a LC/MS method has not been described for GFZ, and is the focus of our report.

In terms of other potential drawbacks associated with previously published GFZ methods, several methods have used agents such as the non-steroidal anti-inflammatory drugs, ibuprofen and celecoxib, as the internal standards (Hengy et al., 1985; Gonzalez-Penas et al., 2001; Niemi et al., 2003; Vittal et al., 2006). In clinical trials, there is a high likelihood that patients may be taking one of these agents, particularly over-the-counter ibuprofen, as part of their clinical care. As such, use of these internal standards in the analytical method may result in assay failure as a result of variable internal standard responses between patients. In the validation of our LC/MS method, we chose to use a commercially available deuterated (d6-substituted) GFZ compound as our internal standard. As the compound is chemically equivalent to the target compound (outside of the deuteration sites), it will be prone to the same matrix effects as the target compound, and will extract from the matrix in a chemically identical manner (Viswanathan et al., 2007). In addition, this is an advantage over previously published LC-MS/MS methods which do not utilize an isotopically-labeled internal standard (Roadcap et al., 2003; Busse et al., 2009).

In the report below, we describe the validation of a LC/MS method for the determination of GFZ concentrations in human plasma. The goal of this method was to combine good sensitivity and specificity, relatively simple sample preparation, and short run times, utilizing single quadrupole mass spectrometry. Additionally, we have used commercially available deuterated-GFZ as the internal standard to avoid possible assay failure due to 
patient consumption of other medications. Finally, we used the validated LC/MS method to determine plasma GFZ concentrations in a clinical pharmacokinetic drug-drug interaction study of GFZ and pioglitazone (an oral antidiabetic agent and CYP2C8 substrate) in healthy volunteers.

\section{Experimental}

\section{Chemicals and Reagents}

GFZ (99.7\% pure) was obtained from the United States Pharmacopeia (Rockville, MD, USA) for use as a reference standard. The internal standard deuterated GFZ (GFZ-d6, 98\% pure), gemfibrozil 1-O- $\beta$-glucuronide (GFZ-gluc), and pioglitazone were purchased from Toronto Research Chemicals Inc (Ontario, Canada). Ammonium acetate (A.C.S. certified, 98\% pure) was purchased from Sigma-Aldrich (St. Louis, MO, USA), and HPLC grade acetonitrile-0.1\% formic acid was purchased from Mallinckrodt Baker, Inc (Phillipsburg, NJ, USA). HPLC grade methanol and formic acid were bought from Fisher Scientific (Fairlawn, NJ, USA). Ultrapure (UP) water was prepared from deionized water in a Barnstead Nanopure System (Thermo Fisher Scientific, Waltham, MA, USA). Plasma with EDTA as an anticoagulant was acquired from Biological Specialty Corporation (Colmar, PA, USA).

\section{LC/MS Instrumentation and Conditions}

The HPLC system utilized was a Surveyor LC autosampler and LC pump (Thermo Fisher, San Jose, CA, USA). The analytical column was a $2.1 \mathrm{~mm} \times 50 \mathrm{~mm}, 5 \mu \mathrm{m}$ Sunfire C18 column (Waters Corporation, Milford, MA, USA). The mobile phase was $0.1 \%$ formic acid in 50:50 acetonitrile/water (v/v) and was delivered at a flow rate of $300 \mu \mathrm{L} / \mathrm{min}$. The analytical column was maintained at $40^{\circ} \mathrm{C}$ resulting in a system backpressure of approximately $500 \mathrm{psi}$. The autosampler needle was washed with a filtered 50:50 methanol:water (v/v) mixture between each injection. A single quadrupole Thermo Fisher MSQ (Thermo Fisher, San Jose, CA, USA) was used in ESI, positive polarity mode. Analytes were detected using single ion monitoring (SIM) mode, with GFZ and GFZ-d6 detected at $m / z$ of 251.51 and 257.56 , respectively. The needle voltage was set at $2.5 \mathrm{kV}$, the cone voltage at $50 \mathrm{~V}$, and the capillary temperature maintained at $535^{\circ} \mathrm{C}$. Nitrogen was used as the source gas, and was maintained at 75 psi. Data acquisition, processing, and storage were performed using Xcalibur software, version 1.3 (Thermo Fisher, San Jose, CA, USA). Calculations were based on peak area ratios of analyte to internal standard. Concentrations are interpolated from a linear least squares regression calibration curve, based on 1/ concentration weighting.

\section{Preparation of Calibration Standards, Internal Standard, and Quality Controls}

A $1 \mathrm{mg} / \mathrm{mL}$ GFZ stock solution was used to prepare working standard stocks of 5, 10, 25, $50,100,250$, and $500 \mu \mathrm{g} / \mathrm{mL}$. A $32 \mu \mathrm{g} / \mathrm{mL}$ solution of GFZ-d6 was prepared for use as a working internal standard stock solution. All GFZ and GFZ-d6 stock solutions were prepared in $100 \%$ methanol and stored in aluminum foil wrapped glass vials at $-20^{\circ} \mathrm{C}$. GFZ quality controls (QC) of $0.5 \mu \mathrm{g} / \mathrm{mL}$ (lower limit of quantification, LLOQ), $1.5 \mu \mathrm{g} / \mathrm{mL}$ (low QC), $10 \mu \mathrm{g} / \mathrm{mL}$ (medium QC), and $40 \mu \mathrm{g} / \mathrm{mL}$ (high QC) were prepared in plasma and stored at $-80^{\circ} \mathrm{C}$.

\section{Sample Preparation}

Plasma GFZ standards were prepared in microcentrifuge tubes on a daily basis by spiking $12.5 \mu \mathrm{L}$ of the correct GFZ working standard stock solution into $125 \mu \mathrm{L}$ of blank plasma resulting in plasma GFZ standard (calibration) concentrations of 0.5, 1.0, 2.5, 5.0, 10.0, 
25.0, and $50.0 \mu \mathrm{g} / \mathrm{ml}$. Patient and QC plasma samples $(125 \mu \mathrm{L})$ were added to microcentrifuge tubes and a $20 \mu \mathrm{L}$ volume of working internal standard stock was then added to every sample, except the double blank. To normalize the sample volume and content between samples, $12.5 \mu \mathrm{L}$ of $100 \%$ methanol was added to the blank sample, QC samples, and patient samples, while $32.5 \mu \mathrm{L}$ of $100 \%$ methanol was added to the double blank sample. A $120 \mu \mathrm{L}$ volume of ammonium acetate $(500 \mathrm{mM})$ was added to each sample and the mixture was vortex mixed. Then, $500 \mu \mathrm{L}$ of acetonitrile (containing $0.1 \%$ formic acid) was added to each sample and mixed thoroughly by vortexing. Each tube was centrifuged at $9200 \times \mathrm{g}$ for $10 \mathrm{~min}$, producing a protein pellet at the bottom. The supernatant from each tube was diluted two-fold with ultrapure water, and a $15 \mu \mathrm{L}$ aliquot of this sample was injected onto the LC/MS system. The resulting retention times for both GFZ and GFZd6 were approximately 5 minutes.

\section{Method Validation}

Validation of the method included an evaluation of the following characteristics: assay accuracy and precision, dilution accuracy and precision, recovery, calibration curve performance, analyte stability, specificity/selectivity, and matrix effects. These validation experiments followed the FDA Guidance for Bioanalytical Method Validation (Food and Drug Administration, 2001). Accuracy was evaluated by calculating the percent deviation from the nominal concentration, and is reported as relative error (RE) . Per FDA guidelines, the mean value should be within $15 \%$ of the nominal value, except at LLOQ, where it should be within $20 \%$ of the nominal value . Precision was determined by calculating the coefficient of variation (CV) of replicates within one sample run (intra-day) and between sample runs (inter-day). Intra- and inter-day accuracy and precision were determined by the performance of four concentrations of QCs (LLOQ $=0.5 \mu \mathrm{g} / \mathrm{mL}$; low QC $=1.5 \mu \mathrm{g} / \mathrm{mL}$; medium QC $=10 \mu \mathrm{g} / \mathrm{mL}$; and high $\mathrm{QC}=40 \mu \mathrm{g} / \mathrm{mL}$ ). Concentration of the low QC was within three times the LLOQ concentration and the high QC was within $80 \%$ of the upper limit of quantitation (ULOQ). The low QC, medium QC, and high QC were run in five replicates on five separate days. The LLOQ QC was run in five replicates on three separate days. Per FDA guidelines, the precision at each concentration level should not exceed $15 \% \mathrm{CV}$, except for the LLOQ, where it should not exceed $20 \%$ CV . In order to determine the accuracy and precision $(n=3)$ of measuring GFZ in diluted samples, a QC was prepared at a concentration of $100 \mu \mathrm{g} / \mathrm{mL}$, then diluted to $20 \mu \mathrm{g} / \mathrm{mL}$ (5x), and to $10 \mu \mathrm{g} / \mathrm{mL}$ (10x) with blank plasma.

Calibration curve performance was also assessed by evaluating deviation of standards from the nominal concentration and evaluating the slope, intercept, and coefficient of determination $\left(r^{2}\right)$ of the weighted 1/concentration linear regression lines. At least six nonzero standards were required for a valid calibration curve where $\pm 20 \%$ from the nominal value was acceptable at the LLOQ and $\pm 15 \%$ from the nominal value accepted at all other concentrations. If a calibrator did not meet these criteria, it was dropped from the calibration curve and the curve was recalculated.

The stability of GFZ in EDTA plasma was tested by subjecting QCs to different test conditions. The test conditions included five freeze/thaw cycles, five days storage at room temperature, and stability of extracted samples after four days of storage in the autosampler $\left(15^{\circ} \mathrm{C}\right)$. Freeze/thaw stability of the QCs was tested in triplicate at the low QC and high QC levels after five freeze/thaw cycles. The samples were allowed to thaw completely and remained at room temperature for at least one hour. The samples were returned to freezer storage conditions at $-80^{\circ} \mathrm{C}$ for 24 hours prior to removal for the next freeze/thaw cycle. The stability of GFZ in plasma at room temperature was tested for thawed QC samples (medium QC level in triplicate) maintained at room temperature for 5 days prior to extraction and analysis. The GFZ stability of extracted samples was determined by 
analyzing GFZ concentrations from QC extracts (low QC and high QC levels in triplicate) after four days storage in the autosampler at $15^{\circ} \mathrm{C}$. The samples were considered to be stable at a given condition if the mean values obtained from the treated QCs were within $\pm 10 \%$ of the mean values of the untreated or reference QC samples that were run within the same analytical run.

The stability of GFZ and GFZ-d6 stock solutions was analyzed by comparing the signal response of a freshly prepared stock solution sample with the signal response from a preparatory stock solution sample prepared two months earlier. Long term stability of GFZ in plasma was determined by comparing analyte response of a freshly prepared medium QC level $(10 \mu \mathrm{g} / \mathrm{mL}, \mathrm{n}=3)$ to that of a one month old medium QC level.

In terms of assay specificity and selectivity, GFZ is highly metabolized into the glucuronide conjugate, gemfibrozil 1-O- $\beta$ glucuronide (GFZ-gluc). It is possible that GFZ-gluc could degrade into GFZ during ionization in the source, or could co-elute with the parent molecule resulting in an inaccurate determination of GFZ concentrations due to signal enhancement. Another item of consideration was that our method was to be applied to clinical samples from a GFZ-pioglitazone drug-drug interaction study, in which all samples contained the drug, pioglitazone. As such, assay specificity and selectivity for this method were investigated by assessing the effects of GFZ-gluc and pioglitazone on the following: quantitation of GFZ, absence of signal from multiple sources of blank plasma, specificity of the signal in the SIM channels for both GFZ and GFZ-d6 (i.e., channel cross talk), and assessment of carryover. To assess the effects of pioglitazone and GFZ-gluc on the quantitation of GFZ, pioglitazone $2 \mu \mathrm{g} / \mathrm{mL}$ and GFZ-gluc $25 \mu \mathrm{g} / \mathrm{mL}$ were spiked into triplicate medium QCs. The samples were subjected to the sample preparation and analysis as described above and results compared to triplicate reference medium QCs analyzed within the same run. A difference of more than $10 \%$ between the two sets of QCs was considered to be indicative of interference.

Specificity of the GFZ and GFZ-d6 signal from six different sources of blank EDTA plasma were assessed. This was done to show that different plasma sources did not contribute to either the analyte or the internal standard responses. The SIM channels for both GFZ and GFZ-d6 were also monitored for cross-talk. The GFZ SIM channel was monitored for significant response when only GFZ-d6 was present at concentrations consistent with extracted samples and similarly, the GFZ-d6 SIM channel was monitored for significant signal when GFZ was injected at a concentration equal to the ULOQ $(50 \mu \mathrm{g} / \mathrm{mL})$. Carryover from the LC system was also assessed by analyzing a blank plasma sample immediately following an injection of an extracted ULOQ $(50 \mu \mathrm{g} / \mathrm{mL})$ sample.

One of the most important assessments for any MS-derived quantitation method is a proper assessment of the matrix effects associated with the measurement, and if any existing matrix effects may have deleterious effects on analyte quantitation. In order to determine if endogenous compounds in plasma suppressed or enhanced GFZ or GFZ-d6 ionization during the assay, potential matrix effects were tested by comparing five different lots of EDTA plasma (Matuszewski et al., 2003). Three sets of samples (i.e., set 1, set 2, and set 3) were prepared containing GFZ standards of $1 \mu \mathrm{g} / \mathrm{mL}, 5 \mu \mathrm{g} / \mathrm{mL}$, and $25 \mu \mathrm{g} / \mathrm{mL}$ in five different lots of plasma (set 2 and set 3 only). In set 1 samples (neat samples), the analyte and internal standard were added to mobile phase. In set 2 samples, the analyte and internal standard were spiked into the post-extracted plasma sample. In set 3 samples, the analyte and internal standard were spiked into plasma and then extracted as described above. A comparison of set 1 and set 2 samples yielded a quantitative measure of observed matrix effects. A comparison set 2 and set 3 samples demonstrated analyte recovery from the extraction process. The difference between set 1 and set 3 samples described the overall 
efficiency of the analytical process. The effects of the matrix on the assay were determined by comparing the regression line slopes and peak area ratios for each concentration level from the different lots of plasma, as well as by examining the precision of the analyte and internal standard areas and ratios for each sample set and plasma lot.

\section{Clinical Application}

The method was used to analyze plasma samples from healthy volunteers who participated in a GFZ-pioglitazone drug-drug interaction study. The protocol was approved by the Colorado Multiple Institutional Review Board, and all patients gave written informed consent. Subjects were given GFZ $600 \mathrm{mg}$ every 12 hours for 8 doses, with pioglitazone 15 mg administered concomitantly at the fifth dose. At the fifth dose, subjects were admitted to the University of Colorado Clinical Translational Research Center, and blood was collected in EDTA tubes and sampled for GFZ levels pre-dose and 2, 3, 4, 5, 6, 8, and 10 hours postdose. The blood samples were centrifuged and plasma was collected and stored at $-80^{\circ} \mathrm{C}$ until analysis. Plasma concentrations of GFZ were analyzed as described in the method above. GFZ pharmacokinetic parameters were determined by noncompartmental methods using WinNonlin software 5.2.1 (Pharsight Corporation, Mountain View, CA, USA). GFZ plasma concentration-time curves were generated, and the maximum plasma concentration $\left(\mathrm{C}_{\max }\right)$, time to reach maximum plasma concentration $\left(\mathrm{T}_{\max }\right)$, and minimum plasma concentration $\left(\mathrm{C}_{\mathrm{min}}\right)$ were read from these curves. The linear trapezoidal method was used to calculate the dose interval area under the plasma concentration-time $\left(\mathrm{AUC}_{\mathrm{tau}}\right)$. The halflife $\left(t_{1 / 2}\right)$ of GFZ was calculated from the following equation: $t_{1 / 2}=\ln 2 / k_{e}$, where $k_{e}$ was calculated using linear regression analysis of the log-linear portion of the concentration-time curve.

\section{Results and Discussion Chromatography}

The chemical structures of GFZ (MW 250) and GFZ-d6 (MW 256) are shown in Figure 1 and the full scan mass spectra of GFZ and GFZ-d6 are shown in Figure 2. Representative LC/MS chromatograms are shown in Fig.3. Fig.3A. shows a blank plasma sample; Fig.3B. shows a blank plasma sample spiked with GFZ-d6 internal standard; Fig.3C. shows a blank plasma sample spiked with GFZ $0.5 \mu \mathrm{g} / \mathrm{mL}$ (LLOQ) and GFZ-d6 internal standard; Fig.3.D. shows a blank plasma sample spiked with GFZ $50 \mu \mathrm{g} / \mathrm{mL}$ (ULOQ) and GFZ-d6 internal standard; and Fig.3.E. shows a patient sample. In each figure, the top chromatogram shows the GFZ response while the bottom chromatogram shows the GFZ-d6 response. The patient chromatogram (Fig.3.E.) illustrates the importance of having chromatographic separation of the GFZ-gluc from the GFZ peak. The GFZ-gluc peak appears at 1.7 min in the GFZ SIM channel, which is a result of GFZ-gluc having the glucuronide cleaved in the MS ESI source during patient sample analysis. It should be noted that this GFZ-gluc peak was not observed in the non-patient specimens (i.e., spiked standards or QCs), as would be expected. However, the peak at 1.7 min was observed in the spiked GFZ-gluc specificity QM sample as described in the methods section above.

LC/MS conditions were optimized to yield both efficient and sensitive detection of the analytes. Chromatographic conditions were optimized so both GFZ and GFZ-d6 eluted off the column simultaneously within five minutes, but separate from GFZ-gluc. The short run time demonstrates that the above method could easily be used for quantitation of a large number of samples relatively rapidly. Analyte infusion experiments were utilized to optimize MS source conditions and produce an acceptable signal to noise ratio for GFZ at the LLOQ. The LC/MS conditions were such that detection was not only accurate and precise, but selective and specific to the analytes being quantitated. 


\section{Method Validation}

A $1 /$ concentration weighting was used to fit a linear least squares regression calibration curve to the response versus concentration data. Good linearity in the range of $0.5 \mu \mathrm{g} / \mathrm{mL}$ to $50 \mu \mathrm{g} / \mathrm{mL}$ was achieved, with typical $r^{2}$ values between 0.9983 and 0.9998 for the five validation runs. These five validation runs yielded lines with an average slope of $0.133 \pm$ 0.0046 (mean $\pm \mathrm{SD}, \mathrm{n}=5)$ and a y-intercept of $-0.010 \pm 0.0049($ mean $\pm \mathrm{SD}, \mathrm{n}=5)$ with a precision of $3.46 \%$ (Table 1). The LLOQ of the assay was $0.5 \mu \mathrm{g} / \mathrm{mL}$, which is consistent with sensitivities previously reported for HPLC-UV and fluorescence methods (Schneck et al., 2004;Randinitis et al., 1986;Kang et al., 2009;Lilja et al., 2005). No carryover was noted in the studied GFZ concentration range, as blank samples injected after high standard injection showed no detectable peak.

Intra-assay precision and accuracy were determined by analyzing the LLOQ $(0.5 \mu \mathrm{g} / \mathrm{mL}$, $\mathrm{n}=5)$ in three separate analytical runs, and analyzing the low $\mathrm{QC}(1.5 \mu \mathrm{g} / \mathrm{mL}, \mathrm{n}=5)$, medium $\mathrm{QC}(10 \mu \mathrm{g} / \mathrm{mL}, \mathrm{n}=5)$, and high QC $(40 \mu \mathrm{g} / \mathrm{mL}, \mathrm{n}=5)$ in five separate analytical runs. The intra-assay precision (reported as CV\%) did not exceed $10.7 \%$ for the four QC levels, while the intra-assay accuracy (expressed as relative error, RE) did not vary more than $\pm 14.4 \%$ from the expected value. Repeated extraction and analysis of these four QC samples yielded an inter-assay precision between $4.4 \%$ and $7.8 \%$, and an inter-assay accuracy ranging from $89.4 \%$ to $104 \%$ (Table 2). Furthermore, we demonstrated that samples could be accurately and precisely diluted five and ten times their original concentrations (accuracy and precision within $\pm 15 \%$ of expected concentrations, data not shown).

The protein precipitation extraction protocol was easy to follow and quick to perform. It yielded a high recovery, ranging from $85.5 \%$ to $94.0 \%$ for the analyte and $86.7 \%$ to $97.1 \%$ for the internal standard (Table 3), with mean values of $89.6 \%$ for the analyte and $90.7 \%$ for the internal standard. These values were determined by comparing set 2 samples (spiked with analyte post extraction) and set 3 samples (spiked with analyte before extraction) of the matrix effect experiment. The recovery is both high and consistent, indicating that little analyte is lost during the sample preparation process, and does not vary from sample to sample.

The success of any LC/MS quantitative analysis rests in the ability of the method to accurately and precisely quantitate analyte concentrations in the presence of matrix. Matrix effects resulting from endogenous compounds in plasma, either signal suppression or enhancement in the MS source, can potentially affect the accuracy and precision of MS quantitative methodologies. By employing the use of the deuterated internal standard, GFZ$\mathrm{d} 6$, we expected to have little variation due to matrix effects. The ratio of GFZ to GFZ-d6 should be relatively close to $100 \%$ throughout the concentration range and for matrix effect, recovery, and process efficiency determinations. We confirmed that the method was not adversely affected by matrix effects in the quantitation of GFZ from EDTA plasma. The resulting matrix effects were quantitated by comparing the mean response from set 1 (neat samples) to the mean response from set 2 samples (post-extract spiked). Compared to set 1 samples prepared in mobile phase, the addition of the extraction matrix in set 2 samples caused a consistent signal enhancement that averaged $107.7 \%$ and $113.7 \%$ for GFZ and the internal standard, respectively, over the concentration range, resulting in an average analyte to internal standard ratio of $94.8 \%$. (Table 3 ).

The combination of analyte loss due to extraction and signal enhancement in the presence of the extraction matrix resulted in a average process efficiency for the ratio of GFZ to GFZ-d6 of $93.6 \%(\mathrm{CV} \%=1.31)$. This indicates that over the range of concentrations tested, the process efficiency (combined matrix effects and recovery) remained consistent. Finally, we found that the mean $\mathrm{CV} \%$ for the five different lots of plasma used for the matrix effects 
experiment were between $2.5 \%$ and $5.4 \%$, while the regression line slopes for the five plasma matrices had a CV of $2.8 \%$. These data suggest that EDTA plasma collected from different individuals will not adversely affect GFZ quantification.

GFZ stability was determined under a wide variety of conditions. As described above, QC samples were used to show that the analyte in plasma is stable through at least five freeze/ thaw cycles and when kept at room temperature for five days. Extracted GFZ samples were also stable when maintained at $15^{\circ} \mathrm{C}$ in the autosampler for at least four days. The stock solutions of both GFZ and GFZ-d6 were shown to be stable for at least two months when stored at $-20^{\circ} \mathrm{C}$. Finally, plasma samples (i.e., QC samples and patient samples) remained stable for at least one month. During the stability testing, no deterioration of GFZ was observed. For each stability test, treated and control samples were within $\pm 10 \%$ of each other and precision $(n=3)$ was within $\pm 15 \%$.

Assay selectivity was determined in a number of ways. First, every analytical run included a single and double blank sample. The single blank showed that the GFZ-d6 internal standard did not contribute to analyte response (no cross-talk of GFZ-d6 into the GFZ SIM channel), while the double blank showed that no signal came from the sample matrix. Furthermore, we examined the extraction of a sample spiked with the highest concentration GFZ standard $(50.0 \mu \mathrm{g} / \mathrm{mL})$, but without internal standard, and found no signal detected in the GFZ-d6 channel from the high GFZ peak (no cross-talk of GFZ into the GFZ-d6 SIM channel). Analysis of six different sources of blank EDTA plasma did not show an analyte or internal standard peak, indicating that the source of plasma does contribute to analyte or internal standard signal. Specificity was also evaluated by examining the effect of two possible interfering compounds, pioglitazone $(2 \mu \mathrm{g} / \mathrm{mL})$ and GFZ-gluc $(25 \mu \mathrm{g} / \mathrm{mL})$ that were known to exist in the samples of patients participating in our drug-drug interaction study.

Concentrations of pioglitazone and GFZ-gluc were chosen for use in the selectivity experiments based on the highest concentrations that are likely to be observed in patient plasma (Hermening et al., 2000; Deng et al., 2005). Results demonstrated that the mean calculated medium QC GFZ concentrations changed by less than 7\% between the control and the pioglitazone and GFZ-gluc spiked samples, demonstrating method specificity in the presence of these two compounds.

\section{Clinical Application}

Plasma samples from six healthy volunteers participating in a GFZ-pioglitazone drug-drug interaction study were analyzed for GFZ concentrations, as described above. At the time of blood sampling, subjects had received 5 oral doses of GFZ $600 \mathrm{mg}$ every 12 hours. The GFZ plasma concentration-time profile for these subjects is shown in Fig.4. GFZ pharmacokinetic parameters (mean $\pm \mathrm{SD}$ ) for these six subjects were: $\mathrm{C}_{\max }, 31.2 \pm 11.4 \mu \mathrm{g} / \mathrm{mL} ; \mathrm{T}_{\max }, 2.2 \pm$ 0.4 hours; $\mathrm{C}_{\min }, 1.3 \pm 0.47 \mu \mathrm{g} / \mathrm{mL} ; \mathrm{t}_{1 / 2}, 1.8 \pm 0.2$ hours; and $\mathrm{AUC}_{\mathrm{tau}}, 113.1 \pm 37.5 \mu \mathrm{g} * \mathrm{~h} / \mathrm{mL}$.

\section{Conclusion}

A reliable and simple method for determining GFZ concentrations in human plasma by LC/ MS was validated. Assay sensitivity was similar to that observed from previously published HPLC-UV and fluorescence methods, but run times were substantially shorter with this LC/ MS method. Moreover, this LC/MS method utilized a deuterated GFZ internal standard which was shown to provide GFZ results that were free from significant matrix effects. In the clinical setting, this is an advantage over other GFZ analytical methods that used prescription or over-the-counter drugs as the internal standard (Hengy et al., 1985; Gonzalez-Penas et al., 2001; Niemi et al., 2003; Roadcap et al., 2003; Vittal et al., 2006; Busse et al., 2009). 
The $0.5 \mu \mathrm{g} / \mathrm{mL}$ level for the LLOQ of this method easily met the validation requirements, indicating that if necessary, the method could potentially be modified for use at lower concentration levels. Sensitivity could be increased by injecting a larger volume or by drying the supernatant and reconstituting in a lower volume. However, this would not be necessary in most clinical studies, as trough concentrations of GFZ $(600 \mathrm{mg}$, twice daily dosing) are generally above $1 \mu \mathrm{g} / \mathrm{ml}$, as we observed in this study (Schneck et al., 2004). The use of LC/MS, which is less costly and less technically demanding than LC-MS/MS, did not come with a sacrifice in accuracy or precision in comparison to a tandem MS system. The validated method was selective for the desired analyte, and reproducible both within runs and between days. Finally, the method was successfully applied to a clinical protocol. The GFZ pharmacokinetic parameters observed in this study (i.e., $\mathrm{C}_{\max }=31.2 \pm$ $11.4 \mu \mathrm{g} / \mathrm{ml}$ and $\mathrm{AUC}=113.1 \pm 37.5 \mu \mathrm{g} * \mathrm{~h} / \mathrm{ml}$ ) were similar to those reported in studies utilizing other analytical methods (e.g., HPLC-UV, $\mathrm{C}_{\max }=27.7 \pm 8.6 \mu \mathrm{g} / \mathrm{ml}, \mathrm{AUC}=101 \pm 35$ $\mu \mathrm{g} * \mathrm{~h} / \mathrm{ml}$; LC-MS/MS, $\mathrm{C}_{\max }=42.4 \pm 10.8$, AUC $=105 \pm 29 \mu \mathrm{g} * \mathrm{~h} / \mathrm{ml}$ ) (Niemi et al., 2003; Tornio et al., 2008). These data demonstrate that LC/MS is a useful method for the determination of GFZ plasma concentrations in human clinical pharmacology studies, particularly those investigating drug-drug interactions with GFZ utilized as a CYP2C8 or OATP1B1 inhibitor.

\section{Acknowledgments}

The authors wish to thank Peter Anderson, Pharm.D. and Uday Kompella, Ph.D. for the use of their laboratory equipment and resources. This work was supported, in part, by National Institutes of Health Grant K23 DK073197 (CLA) and M01 RR000051 (University of Colorado Denver Clinical Translational Research Center).

Funding Source: This work was supported, in part, by a grant from the National Institutes of Health (K23 DK073197 to CLA).

\section{Abbreviations}

CYP2C8

CV

GFZ

GFZ-d6

GFZ-gluc

LC/MS

LC-MS/MS

LLOQ

OATP1B1

QC

RE

ULOQ cytochrome P450 2C8

coefficient of variation

gemfibrozil

gemfibrozil-d6

gemfibrozil 1-O- $\beta$-glucuronide

single quadrupole mass spectrometry

tandem mass spectrometry

lower limit of quantification

organic anion-transporting polypeptide 1B1

quality control

relative error

upper limit of quantification

\section{References}

Backman JT, Kyrklund C, Neuvonen M, Neuvonen PJ. Gemfibrozil greatly increases plasma concentrations of cerivastatin. Clin Pharmacol Ther. 2002; 72:685-691. [PubMed: 12496749] 
Busse KH, Hadigan C, Chairez C, Alfaro RM, Formentini E, Kovacs JA, Penzak SR. Gemfibrozil Concentrations Are Significantly Decreased in the Presence of Lopinavir-Ritonavir. J Acquir Immune Defic Syndr. 2009

Deng LJ, Wang F, Li HD. Effect of gemfibrozil on the pharmacokinetics of pioglitazone. Eur J Clin Pharmacol. 2005; 61:831-836. [PubMed: 16283275]

Food and Drug Administration. Guidance for Industry: Bioanalytical Method Validation. US Department of Health and Human Services, FDA, Center for Drug Evaluation and Research; Rockville, MD: 2001. http://www.fda.gov/downloads/Drugs/GuidanceComplianceRegulatoryInformation/Guidances/ UCM070107.pdf. Accessed 09/24/09

Forland SC, Chaplin L, Cutler RE. Assay of gemfibrozil in plasma by "high-performance" liquid chromatography. Clin Chem. 1987; 33:1938. [PubMed: 3478157]

Gonzalez-Penas E, Agarraberes S, Lopez-Ocariz A, Garcia-Quetglas E, Campanero MA, Carballal JJ, Honorato J. A sensitive method for the determination of gemfibrozil in human plasma samples by RP-LC. J Pharm Biomed Anal. 2001; 26:7-14. [PubMed: 11451637]

Hengy H, Kolle EU. Determination of gemfibrozil in plasma by high performance liquid chromatography. Arzneimittelforschung. 1985; 35:1637-1639. [PubMed: 3867353]

Hermening A, Grafe AK, Baktir G, Mutschler E, Spahn-Langguth H. Gemfibrozil and its oxidative metabolites: quantification of aglycones, acyl glucuronides, and covalent adducts in samples from preclinical and clinical kinetic studies. J Chromatogr B Biomed Sci Appl. 2000; 741:129-144. [PubMed: 10872583]

Jaakkola T, Backman JT, Neuvonen M, Neuvonen PJ. Effects of gemfibrozil, itraconazole, and their combination on the pharmacokinetics of pioglitazone. Clin Pharmacol Ther. 2005; 77:404-414. [PubMed: 15900286]

Kang X, Wang F, Xie Z, Li H. A high performance liquid chromatography method for simultaneous determination of rosiglitazone and gemfibrozil in human plasma. J Chromatogr B Analyt Technol Biomed Life Sci. 2009; 877:645-648.

Lilja JJ, Backman JT, Neuvonen PJ. Effect of gemfibrozil on the pharmacokinetics and pharmacodynamics of racemic warfarin in healthy subjects. Br J Clin Pharmacol. 2005; 59:433439. [PubMed: 15801938]

Lopid Prescribing Information. Pfizer, Inc; November. 2008 Available at: http://media.pfizer.com/files/products/uspi_lopid.pdf

Matuszewski BK, Constanzer ML, Chavez-Eng CM. Strategies for the assessment of matrix effect in quantitative bioanalytical methods based on HPLC-MS/MS. Anal Chem. 2003; 75:3019-3030. [PubMed: 12964746]

McIntosh MP, Carlson BJ, Schorno KS, Rajewski RA. Single quadrupole mass spectrometry for preclinical pharmacokinetic analysis: quantitation of carvedilol in dog plasma. J Chromatogr B Analyt Technol Biomed Life Sci. 2007; 852:665-668.

Nakagawa A, Shigeta A, Iwabuchi H, Horiguchi M, Nakamura K, Takahagi H. Simultaneous determination of gemfibrozil and its metabolites in plasma and urine by a fully automated high performance liquid chromatographic system. Biomed Chromatogr. 1991; 5:68-73. [PubMed: 1868260]

Nakagomi-Hagihara R, Nakai D, Tokui T, Abe T, Ikeda T. Gemfibrozil and its glucuronide inhibit the hepatic uptake of pravastatin mediated by OATP1B1. Xenobiotica. 2007; 37:474-486. [PubMed: 17523051]

Niemi M, Backman JT, Granfors M, Laitila J, Neuvonen M, Neuvonen PJ. Gemfibrozil considerably increases the plasma concentrations of rosiglitazone. Diabetologia. 2003; 46:1319-1323. [PubMed: 12898007]

Niemi M, Backman JT, Neuvonen M, Neuvonen PJ. Effects of gemfibrozil, itraconazole, and their combination on the pharmacokinetics and pharmacodynamics of repaglinide: potentially hazardous interaction between gemfibrozil and repaglinide. Diabetologia. 2003; 46:347-351. [PubMed: 12687332]

Ogilvie BW, Zhang D, Li W, Rodrigues AD, Gipson AE, Holsapple J, Toren P, Parkinson A. Glucuronidation converts gemfibrozil to a potent, metabolism-dependent inhibitor of CYP2C8: 
implications for drug-drug interactions. Drug Metab Dispos. 2006; 34:191-197. [PubMed: 16299161]

Prueksaritanont T, Richards KM, Qiu Y, Strong-Basalyga K, Miller A, Li C, Eisenhandler R, Carlini EJ. Comparative effects of fibrates on drug metabolizing enzymes in human hepatocytes. Pharm Res. 2005; 22:71-78. [PubMed: 15771232]

Randinitis EJ, Parker TD 3rd, Kinkel AW. Liquid chromatographic determination of gemfibrozil and its metabolite in plasma. J Chromatogr. 1986; 383:444-448. [PubMed: 3470298]

Roadcap BA, Musson DG, Rogers JD, Zhao JJ. Sensitive method for the quantitative determination of gemfibrozil in dog plasma by liquid-liquid cartridge extraction and liquid chromatography-tandem mass spectrometry. J Chromatogr B Analyt Technol Biomed Life Sci. 2003; 791:161-170.

Schneck DW, Birmingham BK, Zalikowski JA, Mitchell PD, Wang Y, Martin PD, Lasseter KC, Brown CD, Windass AS, Raza A. The effect of gemfibrozil on the pharmacokinetics of rosuvastatin. Clin Pharmacol Ther. 2004; 75:455-463. [PubMed: 15116058]

Shitara Y, Hirano M, Sato H, Sugiyama Y. Gemfibrozil and its glucuronide inhibit the organic anion transporting polypeptide 2 (OATP2/OATP1B1:SLC21A6)-mediated hepatic uptake and CYP2C8mediated metabolism of cerivastatin: analysis of the mechanism of the clinically relevant drugdrug interaction between cerivastatin and gemfibrozil. J Pharmacol Exp Ther. 2004; 311:228-236. [PubMed: 15194707]

Tornio A, Niemi M, Neuvonen M, Laitila J, Kalliokoski A, Neuvonen PJ, Backman JT. The effect of gemfibrozil on repaglinide pharmacokinetics persists for at least $12 \mathrm{~h}$ after the dose: evidence for mechanism-based inhibition of CYP2C8 in vivo. Clin Pharmacol Ther. 2008; 84:403-411. [PubMed: 18388877]

Viswanathan CT, Bansal S, Booth B, DeStefano AJ, Rose MJ, Sailstad J, Shah VP, Skelly JP, Swann PG, Weiner R. Quantitative bioanalytical methods validation and implementation: best practices for chromatographic and ligand binding assays. Pharm Res. 2007; 24:1962-1973. [PubMed: 17458684]

Vittal S, Shitut NR, Kumar TR, Vinu MC, Mullangi R, Srinivas NR. Simultaneous quantitation of rosuvastatin and gemfibrozil in human plasma by high-performance liquid chromatography and its application to a pharmacokinetic study. Biomed Chromatogr. 2006; 20:1252-1259. [PubMed: 16838266]

Yamazaki M, Li B, Louie SW, Pudvah NT, Stocco R, Wong W, Abramovitz M, Demartis A, Laufer R, Hochman JH, Prueksaritanont T, Lin JH. Effects of fibrates on human organic anion-transporting polypeptide 1B1-, multidrug resistance protein 2- and P-glycoprotein-mediated transport. Xenobiotica. 2005; 35:737-753. [PubMed: 16316932] 
A. Gemfibrozil, MW: 250<smiles>Cc1ccc(C)c(OCCCC(C)(C)C(=O)O)c1</smiles>

B. Gemfibrozil-d6, MW: 256<smiles>Cc1ccc(C)c(OCCCC(C)(C)C(=O)O)c1</smiles>

Figure 1.

Structures of (A) gemfibrozil, and (B) gemfibrozil-d6, internal standard. 

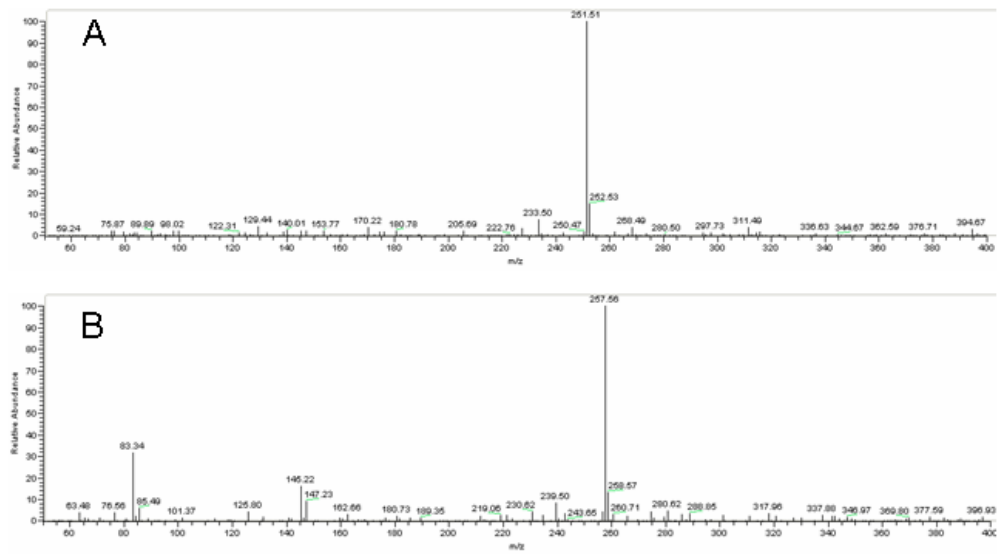

Figure 2.

Full scan mass spectra of (A) gemfibrozil $(\mathrm{m} / \mathrm{z} 251.51)$, and (B) gemfibrozil-d6 $(\mathrm{m} / \mathrm{z}$ 257.56). 

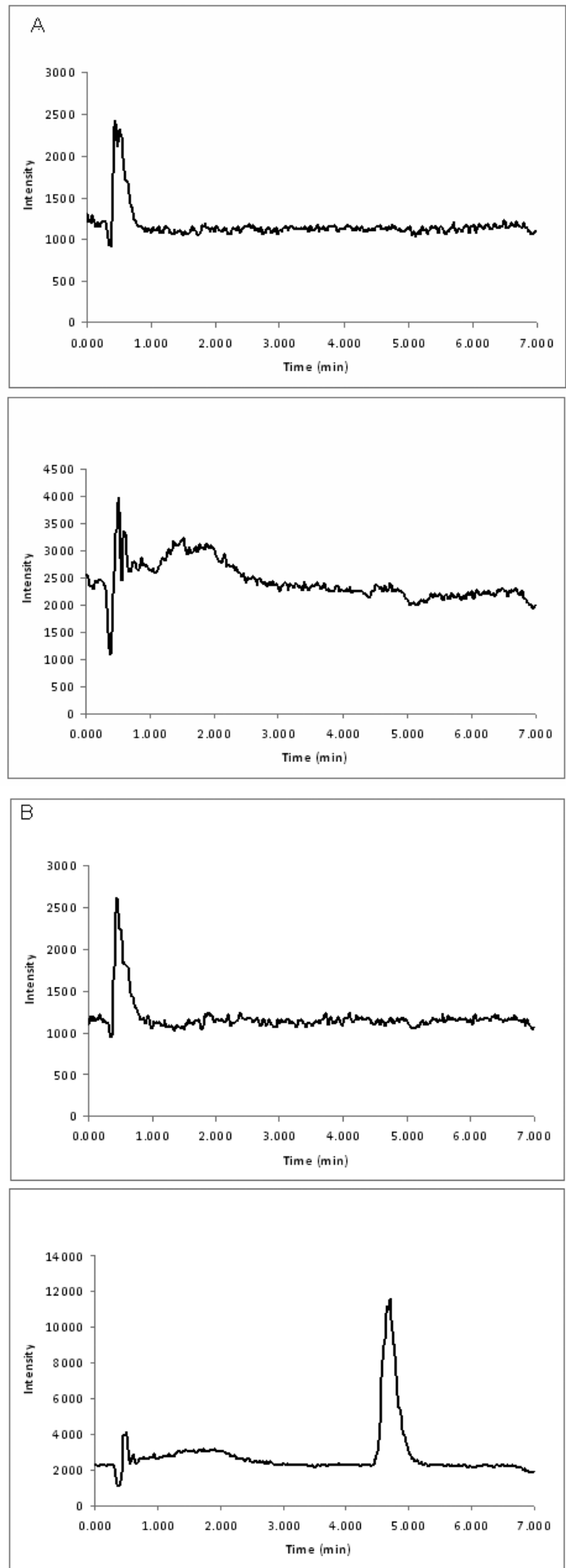

Biomed Chromatogr. Author manuscript; available in PMC 2011 December 1. 

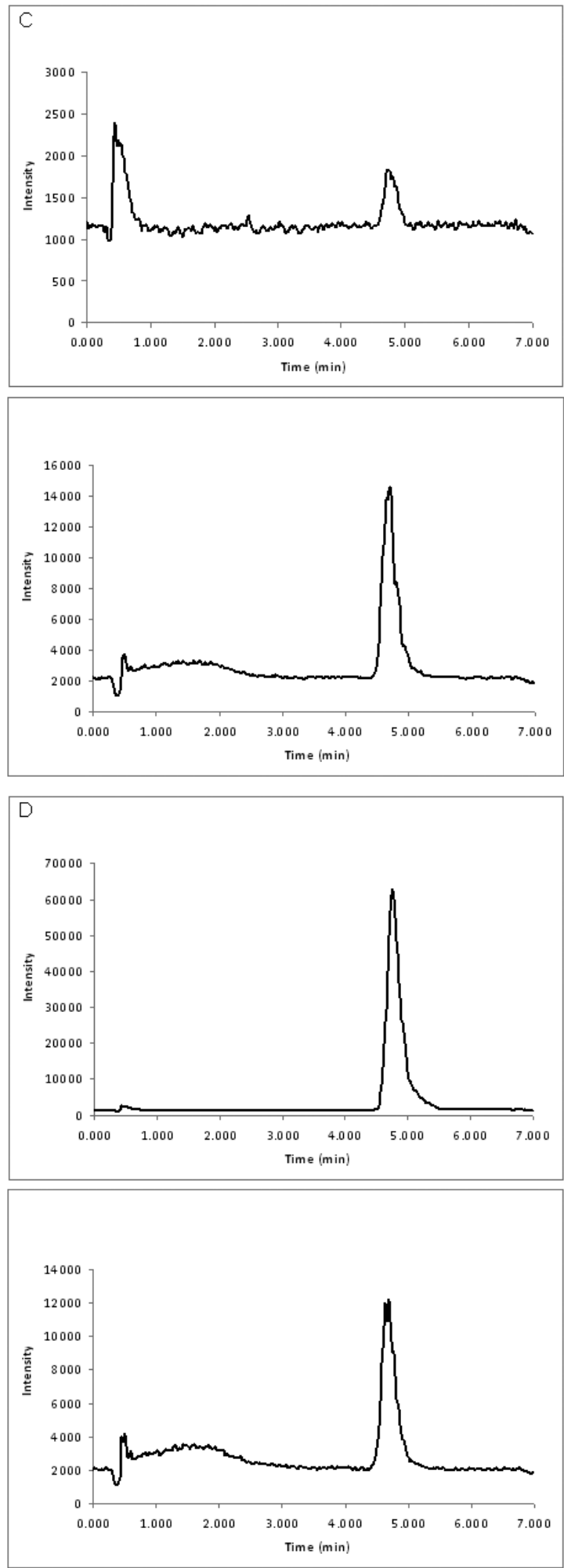

Biomed Chromatogr. Author manuscript; available in PMC 2011 December 1. 

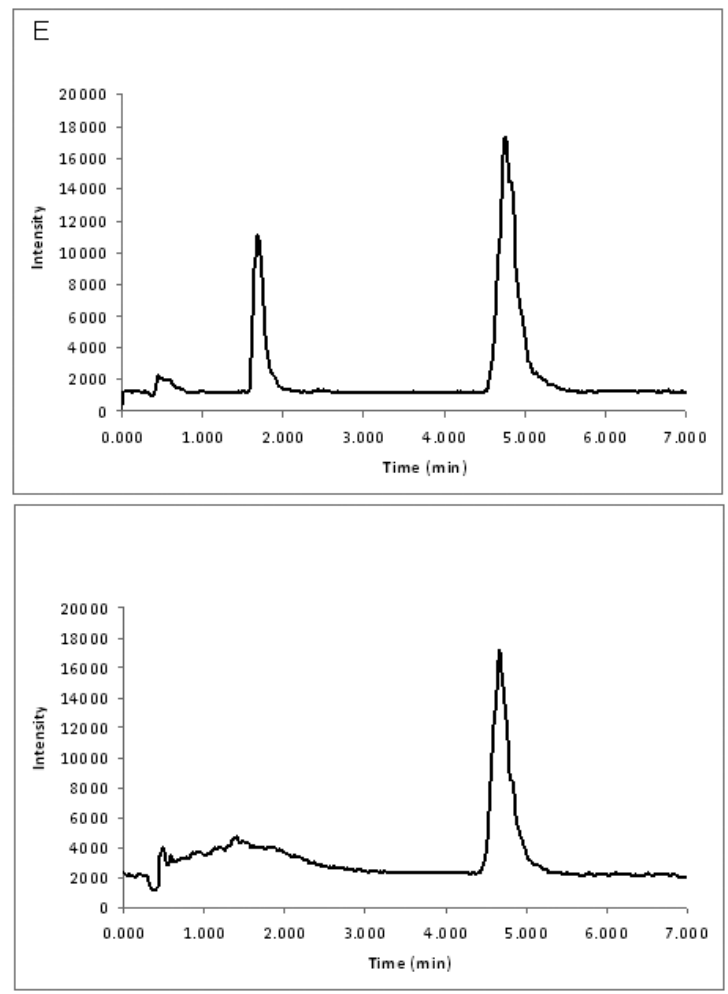

Figure 3.

Representative LC/MS chromatograms. (A) blank plasma sample; (B) blank plasma sample spiked with GFZ-d6 internal standard; (C) blank plasma sample spiked with GFZ LLOQ $(0.5 \mu \mathrm{g} / \mathrm{mL})$ and GFZ-d6 internal standard; (D) blank plasma sample spiked with GFZ ULOQ $(50 \mu \mathrm{g} / \mathrm{mL})$ and GFZ-d6 internal standard; and (E) a patient sample. The top chromatogram is the analyte while the bottom chromatogram is the GFZ-d6 internal standard. The patient sample (E) shows a peak retained at 1.7 minutes, which is due to the presence of a GFZ glucuronide metabolite in vivo, which is cleaved in the MS ESI source. 


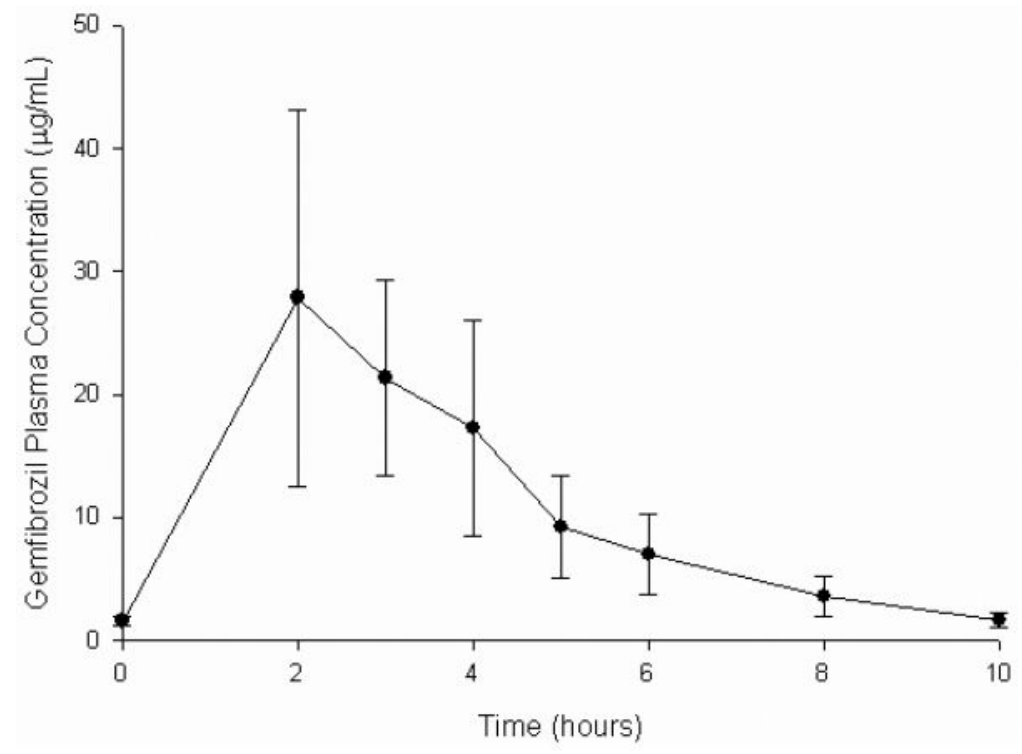

Figure 4.

GFZ plasma concentration-time profile after multiple oral doses of GFZ $600 \mathrm{mg}$ in six healthy volunteers. Data are shown as mean \pm SD at each time point. 
Table 2

GFZ Intra-and inter-assay precision and accuracy

\begin{tabular}{|c|c|c|c|c|}
\hline \multicolumn{5}{|c|}{ Intra-assay precision and accuracy } \\
\hline & $\begin{array}{c}\text { LLOQ } \\
0.5 \mu \mathrm{g} / \mathrm{mL}\end{array}$ & $\begin{array}{c}\text { Low QC } \\
1.5 \mu \mathrm{g} / \mathrm{mL}\end{array}$ & $\underset{10 \mu \mathrm{g} / \mathrm{mL}}{\text { Medium QC }}$ & $\begin{array}{l}\text { High QC } \\
40 \mu \mathrm{g} / \mathrm{mL}\end{array}$ \\
\hline \multicolumn{5}{|l|}{ Run 1} \\
\hline Mean & 0.44 & 1.44 & 10.21 & 41.69 \\
\hline SD & 0.02 & 0.03 & 0.16 & 1.48 \\
\hline CV (\%) & 3.4 & 1.9 & 1.6 & 3.6 \\
\hline $\mathrm{RE}(\%)$ & -12.0 & -4.0 & 2.1 & 4.2 \\
\hline$N$ & 5 & 5 & 5 & 5 \\
\hline \multicolumn{5}{|l|}{ Run 2} \\
\hline Mean & 0.47 & 1.41 & 9.79 & 39.44 \\
\hline SD & 0.05 & 0.03 & 0.28 & 2.44 \\
\hline $\mathrm{CV}(\%)$ & 10.7 & 2.1 & 2.9 & 6.2 \\
\hline $\mathrm{RE}(\%)$ & 5.4 & -5.9 & -2.1 & -1.4 \\
\hline$N$ & 5 & 5 & 5 & 5 \\
\hline \multicolumn{5}{|l|}{ Run 3} \\
\hline Mean & 0.43 & 1.31 & 9.51 & 40.64 \\
\hline SD & 0.01 & 0.03 & 0.18 & 1.33 \\
\hline $\mathrm{CV}(\%)$ & 1.6 & 2.6 & 1.9 & 3.3 \\
\hline $\operatorname{RE}(\%)$ & -14.4 & -12.6 & -4.9 & 1.6 \\
\hline$N$ & 5 & 5 & 5 & 5 \\
\hline \multicolumn{5}{|l|}{ Run 4} \\
\hline Mean & - & 1.53 & 10.17 & 42.68 \\
\hline SD & - & 0.11 & 0.52 & 1.65 \\
\hline $\mathrm{CV}(\%)$ & - & 7.3 & 5.1 & 3.9 \\
\hline $\operatorname{RE}(\%)$ & - & 1.7 & 1.7 & 6.7 \\
\hline$N$ & - & 5 & 5 & 5 \\
\hline \multicolumn{5}{|l|}{ Run 5} \\
\hline Mean & - & 1.38 & 9.45 & 43.47 \\
\hline SD & - & 0.06 & 0.25 & 0.92 \\
\hline $\mathrm{CV}(\%)$ & - & 4.1 & 2.7 & 2.1 \\
\hline $\operatorname{RE}(\%)$ & - & -7.9 & -5.5 & 8.7 \\
\hline$N$ & - & 4 & 5 & 5 \\
\hline \multicolumn{5}{|c|}{ Inter-assay precision and accuracy } \\
\hline & $\begin{array}{c}\text { LLOQ } \\
0.5 \mu \mathrm{g} / \mathrm{mL}\end{array}$ & $\begin{array}{c}\text { Low QC } \\
1.5 \mu \mathrm{g} / \mathrm{mL}\end{array}$ & $\begin{array}{c}\text { Medium QC } \\
10 \mu \mathrm{g} / \mathrm{mL}\end{array}$ & $\begin{array}{c}\text { High QC } \\
40 \mu \mathrm{g} / \mathrm{mL}\end{array}$ \\
\hline Mean & 0.45 & 1.42 & 9.83 & 41.58 \\
\hline SD & 0.04 & 0.09 & 0.43 & 2.09 \\
\hline $\mathrm{CV}(\%)$ & 7.8 & 6.5 & 4.4 & 5.0 \\
\hline RE $(\%)$ & -10.6 & -5.7 & -1.8 & 4.0 \\
\hline
\end{tabular}




\begin{tabular}{|c|c|c|c|c|}
\hline \multicolumn{5}{|c|}{ Intra-assay precision and accuracy } \\
\hline & $\begin{array}{c}\text { LLOQ } \\
0.5 \mu \mathrm{g} / \mathrm{mL}\end{array}$ & $\begin{array}{c}\text { Low QC } \\
1.5 \mu \mathrm{g} / \mathrm{mL}\end{array}$ & $\begin{array}{c}\text { Medium QC } \\
10 \mu \mathrm{g} / \mathrm{mL}\end{array}$ & $\begin{array}{c}\text { High QC } \\
40 \mu \mathrm{g} / \mathrm{mL}\end{array}$ \\
\hline$N$ & 15 & 24 & 25 & 25 \\
\hline
\end{tabular}

LLOQ, lower limit of quantification; $\mathrm{QC}$, quality control; SD, standard deviation; CV, coefficient of variation (measure of precision); RE, relative error (measure of accuracy) 


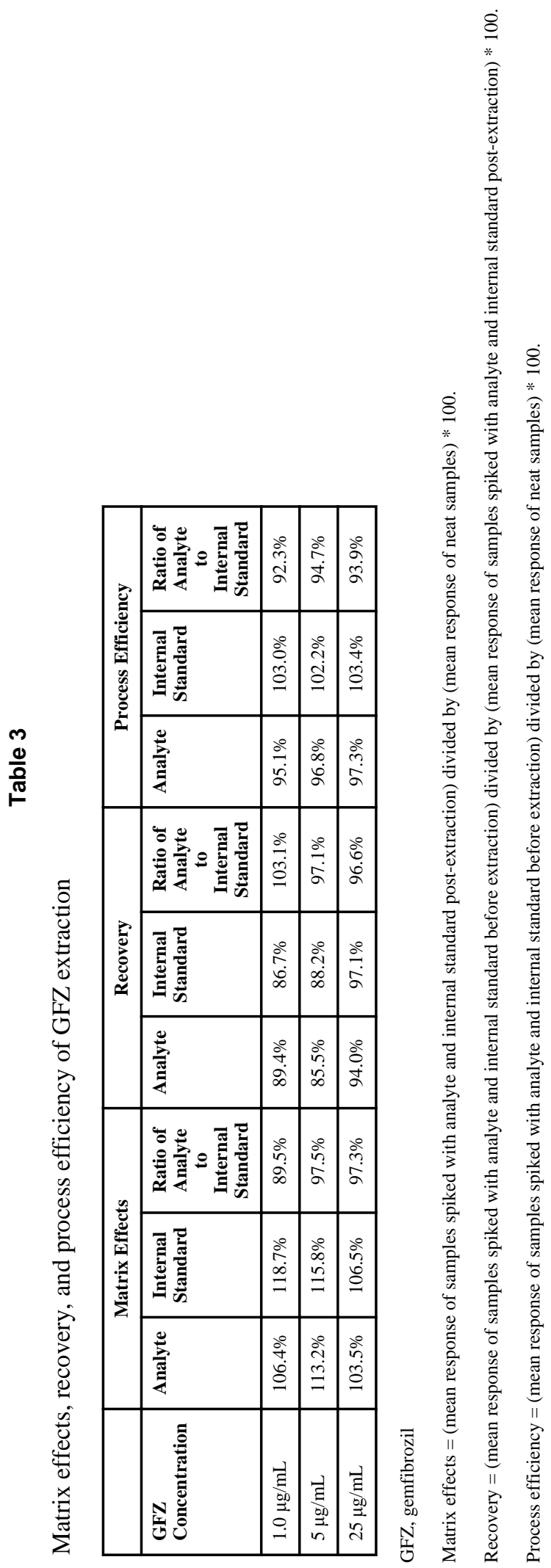

Biomed Chromatogr. Author manuscript; available in PMC 2011 December 1. 